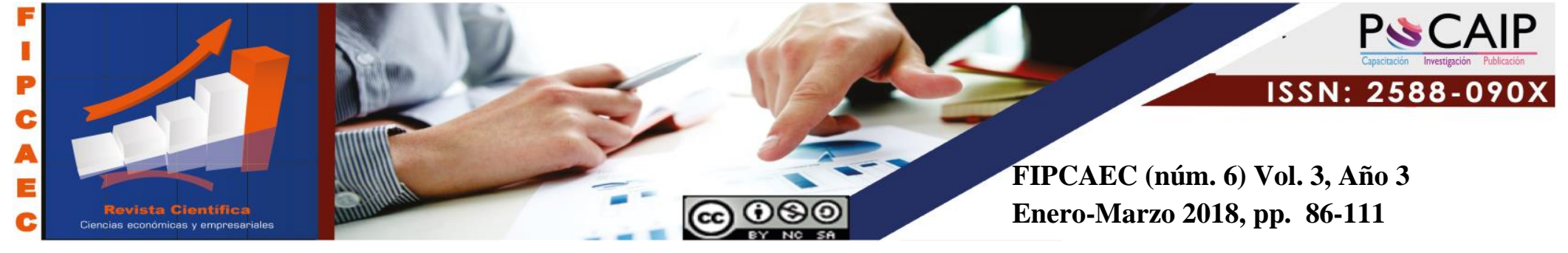

DOI 10.23857/fipcaec.v3i6.68

\title{
La Representación Social de la Economía Socialista en Venezuela
}

The Social Representation of the Socialist Economy in Venezuela as A representação social da economia socialista na Venezuela

\author{
José Gregorio Wagner-Albujas ${ }^{1}$ \\ gregoriowagner@hotmail.com \\ https://orcid.org/0000-0002-5019-1760
}

Correspondencia: gregoriowagner@ hotmail.com

* Recepción: 05/ 10/ 2017 * Aceptación: 12/12/ 2017 *Publicación: 15 /01/ 2018

1 Profesor Evaluador de Administración, Instituto Tecnológico Superior CEMLAD, Quito, Ecuador. 


\section{Resumen}

Este estudio tuvo como finalidad de velar la representación social existente sobre la economía socialista y conocer las expectativas creadas por su implementación, por ello se trazó como objetivo de investigación la descripción de los supuestos y percepciones presentes en las representaciones sociales iniciales de la economía socialista y el determinar cuáles fueron las expectativas generadas por la implementación de la economía socialista en Venezuela. La investigación tuvo como soporte la concepción dialéctica crítica de la sociedad que mira la transición como la totalidad en movimiento real siendo empleado el enfoque cualitativo de investigación, basado en la teoría fundamentada para ayudar a reconocer dentro de la compleja totalidad del entramado de relaciones sociales, la sustantividad de las representaciones sociales de la Economía Socialista, así como las expectativas creadas por su implementación. Los aspectos descritos nos permiten decir que la representación social de la Economía Socialista se presenta en un nivel superficial, ya que algunas de las determinaciones esenciales que forman parte de la construcción socialista y de su economía no son referenciadas de manera precisa. En relación a lo que esperan los diversos actores sociales por la implementación de la economía socialista dentro del cambio epocal en Venezuela, esta debe orientarse a proveer los satisfactores básicos para la sociedad, si no existen satisfactorios básicos, ninguna acción o discurso por muy esperanzador puede funcionar, colocando cada vez más cuesta arriba la construcción de la Economía Socialista.

Palabras Claves: Imaginarios; representaciones sociales; economía socialista; concepción Crítica Dialéctica; cambio epocal.

\section{Abstract}

The purpose of this study was to ensure the existing social representation of the socialist economy and to know the expectations created by its implementation, which is why the description of the assumptions and perceptions present in the initial social representations of the socialist economy and to determine what were the expectations generated by the implementation of the socialist economy in Venezuela. The research was supported by the critical dialectical conception of society that looks at the transition as the totality in real movement, using the 
qualitative research approach, based on the theory based to help recognize within the complex totality of the social relations framework, the substantivity of the social representations of the Socialist Economy, as well as the expectations created by its implementation. The aspects described allow us to say that the social representation of the Socialist Economy is presented on a superficial level, since some of the essential determinations that are part of the socialist construction and its economy are not accurately referenced. In relation to what the various social actors expect for the implementation of the socialist economy within the epochal change in Venezuela, it must be oriented to provide the basic satisfactors for society, if there are no basic satisfactions, no action or speech however hopeful can function, placing more and more uphill the construction of the Socialist Economy.

Keywords: Imaginary; social representations; socialist economy; Dialectical Critical conception; epochal change.

\section{Resumo}

O objetivo deste estudo foi garantir a representação social existente da economia socialista e conhecer as expectativas criadas por sua implementação, razão pela qual a descrição dos pressupostos e percepções presentes nas representações sociais iniciais da economia socialista e determinar quais eram as expectativas geradas pela implementação da economia socialista na Venezuela. A pesquisa foi apoiada pela concepção dialética crítica da sociedade, que considera a transição como a totalidade do movimento real, usando a abordagem qualitativa da pesquisa, baseada na teoria baseada em ajudar a reconhecer dentro da totalidade complexa da estrutura de relações sociais, a substantividade das representações sociais da Economia Socialista, bem como as expectativas criadas por sua implementação. Os aspectos descritos permitem dizer que a representação social da Economia Socialista é apresentada em um nível superficial, uma vez que algumas das determinações essenciais que fazem parte da construção socialista e sua economia não são referenciadas com precisão. Em relação ao que os vários atores sociais esperam para a implementação da economia socialista na época da mudança na Venezuela, deve ser orientado a fornecer os satisfatores básicos para a sociedade, se não houver satisfações básicas, nenhuma ação ou discurso, por mais esperançoso que seja. Cada vez mais difícil a construção da Economia Socialista. 
Palavras-chave: Imaginário; representações sociais; economia socialista; Concepção crítica dialética; mudança de época.

\section{Introducción}

El presente artículo plantea uno de los objetivos propuestos en el trabajo de investigación titulado: Superación de la representación social inicial de la economía socialista dentro del cambio epocal en Venezuela, bajo esa perspectiva el enfoque se dirige a la revisión de las tareas de investigación que fueron abordadas en el año 2016 con respecto a la descripción de los supuestos y percepciones presentes en las representaciones sociales iniciales de la economía socialista y el determinar cuáles fueron las expectativas generadas por la implementación de la economía socialista en Venezuela.

El punto de partida de esta investigación tuvo que ver con la consideración de la necesidad de la transición de la sociedad venezolana hacia una nueva época histórica y su correspondiente elaboración teórica y procedimental para favorecer en el contexto mundial el avance en su realización e irreversibilidad. La necesidad de la transición epocal se va generando de acuerdo a lo planteado por la dirigencia política como efecto de la crítica social y científica de los desastrosos resultados socioculturales y ecológicos exhibidos por el desarrollo de la forma capitalismo y del milenario sistema del capital que ha llegado hasta el punto de poner en riesgo la vida de la especie humana y las otras especies.

En función de estos planteamientos el objetivo de este estudio es presentar los supuestos y percepciones presentes en las representaciones sociales iniciales de la economía socialista y exponer cuáles fueron las expectativas generadas por la implementación de la economía socialista en Venezuela. En lo tocante a la posibilidad del avance en la irreversibilidad de la transición, luego de la caída de la propuesta del socialismo soviético, merece una nueva reflexión crítica. Por eso pensamos en examinar la posibilidad de la reversibilidad del proceso bolivariano, poniendo el énfasis en las dificultades evidentes que presenta su Economía y la relación de ésta con la elaboración teórica de sus orientaciones, pues fue llamada a construirse como socialista por el Presidente Chávez en diciembre de 2006. 
Valga precisar que los hechos actuales de la economía venezolana fueron señalados como provocados por factores externos e internos. Dentro de los externos están sin duda los efectos de la crisis mundial del sistema del capital y las acciones geopolíticas de los adversarios del proceso bolivariano, la intervención e injerencia de parte de la comunidad internacional, entre otros. Pero dentro de los internos estamos obligados a estudiar, hasta qué punto las representaciones sociales que se tienen sobre la economía socialista son aún incipientes, en términos de Dussely Marx, intuiciones (Ahnung) y por tanto contribuyen poco a la orientación de su marcha como irreversible y por el contrario son fuente de orientaciones prácticas inapropiadas a la dimensión de la tarea histórica y obstáculo al movimiento de la totalidad orgánica llamada transición epocal.

En ese contexto contradictorio, además entendemos que la falta de discusión social y científica acerca de lo que significa y representa el socialismo bolivariano y su economía; el tipo de sociedad y de país que queremos, el manejo de la economía, las políticas implementadas, pueden ser elementos que obstaculicen la formación de la conciencia del sujeto histórico del cambio, vista como la definición y construcción del nuevo sistema económico-político coherente con el cambio de época y ajustado al momento histórico que vive el mundo y la región latinoamericana y caribeña.

La realización de esta investigación tuvo como soporte para su orientación la concepción dialéctica crítica de la sociedad (interpretar para transformar y no para contemplar) que mira la transición como la totalidad en movimiento real, desarrollado de manera no lineal por el sujeto social del cambio. Por eso y como resultado de nuestra reflexión hemos elaborado una matriz ontológica, epistemológica, axiológica y metodológica para interpretar el objeto dinámico de estudio. En coherencia con lo dicho fue empleado el enfoque cualitativo de investigación, basado en la teoría fundamentada para ayudar a reconocer dentro de la compleja totalidad del entramado de relaciones sociales, la sustantividad de las representaciones sociales de la Economía Socialista, insertas en ésta, así como las expectativas creadas por su implementación.

Esta investigación reviste mucha importancia, debido a que el tema es de gran trascendencia y actualidad, ya que el debate de los sistemas económicos se encuentra en un momento histórico muy significativo, dado que en nuestras naciones han venido materializándose conducciones económicas diferenciadas a las prácticas capitalistas y se han llevado a cabo algunas experiencias 
de implantes en materia política y económica con orientaciones y rasgos de intencionalidades socialistas y continua manteniéndose la discusión acerca de los modelos económicos y sus repercusiones en nuestras naciones.

En cuanto a la estructura que compone el presente artículo se ha conformado bajo los parámetros de la estructura IMRYD, introducción, materiales y métodos, resultados y discusión.

\section{Materiales y métodos}

En el caso de nuestro estudio fue empleado un enfoque cualitativo de investigación, en esa dirección se utilizó la concepción dialéctica crítica de Marx para el análisis social (interpretar la realidad para impulsar su transformación), combinada con el diseño de la Teoría Fundamentada como herramienta para la obtención de los datos; ésta es utilizada como uno de los diseños que permite crear propuestas y construcciones teóricas basándose en los datos, su objetivo es la interpretación haciendo uso de la intersubjetividad, mostrándonos una realidad emergente que permitirá develar las representaciones sociales de la economía socialista presentes en los informantes clave, tomando como base la noción del imaginario de la economía socialista, para conocer que yace en su fondo, develándolo como la representación social intuitiva, ambiental, susceptible de ser cambiada en un proceso revolucionario, o sea categorizada, vista en su esencia como un constructo científico y participativo de la Nueva Economía.

La recolección de datos se realizó en los ambientes naturales y cotidianos de los participantes o unidades de análisis, fue aplicada la entrevista cualitativa, específicamente la entrevista en profundidad (abierta), una vez hecha la transcripción de las entrevistas se llevó a cabo el análisis de contenido, desde donde pueden hallarse toda clase de registros de datos, trascripción de discursos, entrevistas, registros de observaciones, videos, documentos, que siendo empleados de manera apropiada nos permiten el acceso al conocimiento y la comprensión en diversos aspectos y fenómenos de la vida social, combinado como el análisis de documentos centrales como son algunos trabajos de Mészáros, Marx y Silva. 
Para la entrevista practicada a los informantes clave, se configuraron tres grupos: Profesionales y profesores de economía, con formación y experiencia en el área, los Funcionarios públicos propulsores de la transición al nuevo modelo económico socialista, con cargos de responsabilidad en la ejecución de directrices en materia de políticas públicas. Así como Actores miembros del poder popular organizado, integrantes y participes en organizaciones de base (consejos comunales y comunas), igualmente se emplearon los lineamientos trazados desde la perspectiva del diseño emergente en Teoría Fundamentada, partiendo de la codificación abierta.

La codificación abierta inicia con la conceptualización, se denotan los fenómenos (ideas o cognemas) y una vez que el investigador los observa y estudia, comienza a examinarlos de forma comparativa con las categorías esenciales y determinaciones descritas en el <Enunciado teórico de entrada> siendo utilizada de manera conjunta con herramientas como matrices de similitud, clasificación en función de la distribución de frecuencias y la contrastación temática, lo cual permitió la elaboración de las respectivas representaciones sociales y conocer la expectativas que se encontraban frente a la economía socialista

\section{Resultados}

Para lograr lo propuesto en el objetivo de investigación: Develar los supuestos y percepciones presentes en las representaciones sociales iniciales de la economía socialista dentro del cambio epocal en Venezuela fueron empleados los insumos derivados de la guía de entrevista bajo los ítems 1, 2 y 4, considerados como los más apropiados para dar respuesta a la primera pregunta orientadora, tal como se muestra en el siguiente cuadro: 
Cuadro 1. Tarea de investigación 1

\begin{tabular}{|c|c|c|}
\hline Tar & nta Orientadora & Guía de Entrevista \\
\hline $\begin{array}{l}\text { Develar los supuestos y } \\
\text { percepciones presentes } \\
\text { en las representaciones } \\
\text { sociales superficiales de } \\
\text { la economía socialista } \\
\text { dentro del cambio epocal } \\
\text { en Venezuela. }\end{array}$ & $\begin{array}{l}\text { 1-¿Cuáles son las } \\
\text { representaciones } \\
\text { sociales sobre economía } \\
\text { socialista reproducidas } \\
\text { por algunos de los } \\
\text { actores intervinientes en } \\
\text { la naciente práctica de la } \\
\text { economía socialista en } \\
\text { Venezuela y cuáles son } \\
\text { los supuestos y } \\
\text { percepciones presentes } \\
\text { en éstas? }\end{array}$ & $\begin{array}{l}\text { İtems } \\
\text { 1. ¿Que entendemos } \\
\text { por socialismo? } \\
\text { 2. ¿Que entendemos } \\
\text { por economía } \\
\text { socialista? } \\
\text { 3. ¿Que debe } \\
\text { producir la economía } \\
\text { socialista }\end{array}$ \\
\hline
\end{tabular}

El procedimiento llevado a cabo para el logro de este objetivo fue la grabación y transcripción de las entrevistas, luego se examinaron de la siguiente manera: en primer lugar, las respuestas se agruparon utilizando matrices de síntesis, se sistematizaron tratándose como unidades de significado que denotan representaciones, posteriormente se clasificaron por medio de análisis de similitud donde se identificaron elementos y "familias" de elementos, es decir, cognemas, de un conjunto dado. Un cognema es una familia de ideas que puede estar representado por una sola palabra o frase. Estos elementos estarán más cerca uno del otro en la representación mientras mayor sea la cantidad de personas que los asocian dentro de su discurso.

En segundo lugar, se crearon matrices de similitud que refieren a la cantidad de veces que un elemento cognema ha sido encontrado en relación a otro elemento. Finalmente, se graficaron aquellas relaciones más intensas entre los cognemas principales. Estas representaciones fueron identificadas en atención a los hallazgos provenientes de cada uno de los informantes clave, (Profesionales y profesores de economía, los funcionarios públicos y los miembros del poder popular organizado). Los resultados encontrados se muestran a continuación 
Nociones Iniciales sobre Economía Socialista. (Profesionales y Profesores de Economía)

Una vez transcritas las entrevistas los resultados expuestos indican su aparición en el discurso de las representaciones sociales como unidades de significado (cognemas) que denotan las intuiciones iniciales:

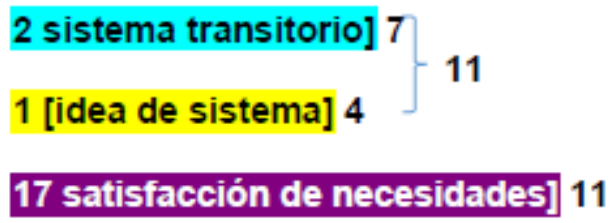

4 distribución equitativa] 10

8 [Busca la igualdad] 9

11 [fomentar la participación] 8

7 [medios de producción públicos 8

3 [medios de producción propiedad del Estado] 7

25 producir bienes y servicios] 7

14 solucionar dificultades 5

30[propiciar el desarrollo endógeno-local] 5

Las intuiciones iniciales aquí presentadas nos aportaron la información necesaria para la construcción de la representación derivada en función de las frecuencias presentadas en el discurso. Esta imagen, llamada "árbol de representación", permite ver las relaciones entre los cognemas centrales destacando las posiciones de cada uno de ellos. 
Representación Social Inicial sobre Economía Socialista. (Profesionales y Profesores de Economía)

Figura 1. Representación Social Inicial sobre Economía Socialista Profesionales y Profesores de Economía

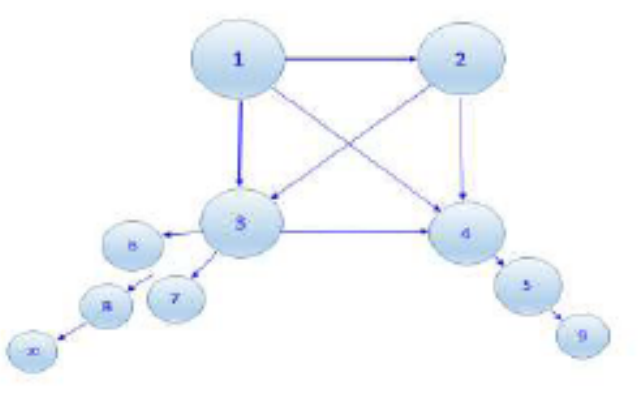

1. Sistema Transitorio

2. Satisfacciones de Necesidades

3. Distribución Equitativa de los Recursos

4. Busca la igualdad

5. Fomentar la Participación

6. Medios de Producción Públicos

7. Medios de Producción Propiedad del Estado

8. Producir Bienes y Servicios

9. Solucionar Dificultades Apremiantes

10. Propiciar el Desarrollo Endógeno-Local

Se entiende como un sistema de carácter transitorio para la satisfacción de necesidades, que promueve una distribución equitativa de los recursos, buscando la igualdad, que fomenta la participación, con medios de producción públicos, y/o medios de producción propiedad del Estado para la producción de bienes y servicios que permitan solucionar las dificultades más apremiantes que presenta la población, propiciando el desarrollo endógeno-local.

Se desprende del análisis de contenido, efectuado en las entrevistas que no se hacen referencias explícitas hacia donde se dirige lo transitorio y a la distribución equitativa del producto social o excedentes, ni al abandono de la división social del trabajo, características esenciales de la Economía Socialista. Con respecto a las relaciones armónicas entre economía con la madre tierra (Pachamama), aspecto fundamental en la praxis de la Economía Socialista su presencia en el discurso la consideramos poco significativa, nucleándose esta representación en torno a cuatro 
concepciones centrales (un sistema transitorio, la satisfacción de necesidades, distribución equitativa de los recursos y búsqueda de la igualdad)

\section{Nociones iniciales sobre Economía Socialista. (Funcionarios públicos)}

- Transcritas las entrevistas, los resultados se muestran cómo se detalla a continuación:

44 debe centrarse en el ser humano 15

5 [búsqueda del bien común] 13

17 satisfacción de necesidades] 11

1 [idea de sistema] 10

47 modelo propio 9

45 mejorar la calidad de vida 9

11 [fomentar la participación] 9

25 producir bienes y servicios] 8

30[propiciar el desarrollo endógeno-local] 7

4 distribución equitativa] 7

46 modelo en construcción 6 


\section{Representación Social Inicial sobre Economía Socialista (Funcionarios Públicos)}

Figura 2. Representación Social Inicial sobre Economía Socialista (Funcionarios Públicos) Discusión de resultados

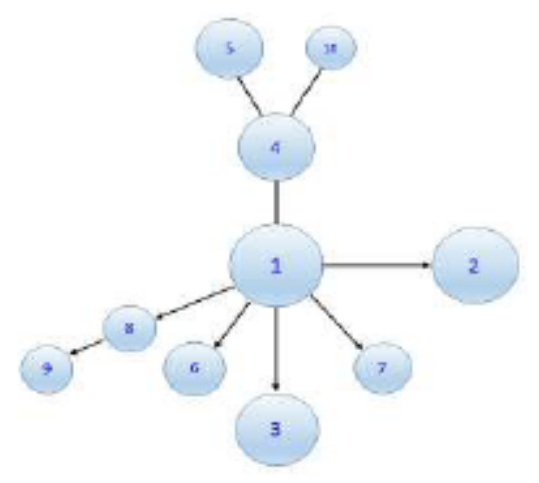

1. Debe centrarse en el ser humano

2. Búsquedas del bien común

3. Satisfacciones de necesidades

4. Idea de sistema

5. Modelo propio

6. Mejorar la calidad de vida

7. Fomentar la participación

8. Producir bienes y servicios

9. Propiciar el desarrollo endógeno-local

10. Modelo en construcción

La Economía Socialista es un proceso que debe centrarse en el ser humano para la búsqueda del bien común y la satisfacción de necesidades de la población constituyéndose como un modelo propio, en fase de construcción, dirigido a mejorar la calidad de vida de las personas, fomentando la participación del pueblo, para producir bienes y servicios que puedan propiciar el desarrollo endógeno-local.

Se aprecia que en las declaraciones procedentes de las entrevistas no se hace referencia de modo preciso a la distribución equitativa del producto social o excedentes, al abandono de la división social del trabajo, ni a que se entiende por mejorar la calidad de vida, bastiones fundamentales de la Economía Socialista. Sobre la armonía que debe existir entre economía y la naturaleza, su 
manifestación en el discurso la observamos limitada. Por su parte el eje discursivo de la Economía Socialista tuvo como foco central la atención del ser humano, acompañado de la búsqueda del bien común y en menor grado la satisfacción de las necesidades.

Nociones Iniciales sobre Economía Socialista. (Miembros del Poder Popular Organizado)

Hecha la sistematización de las entrevistas las nociones iniciales fueron expresadas de la siguiente manera:

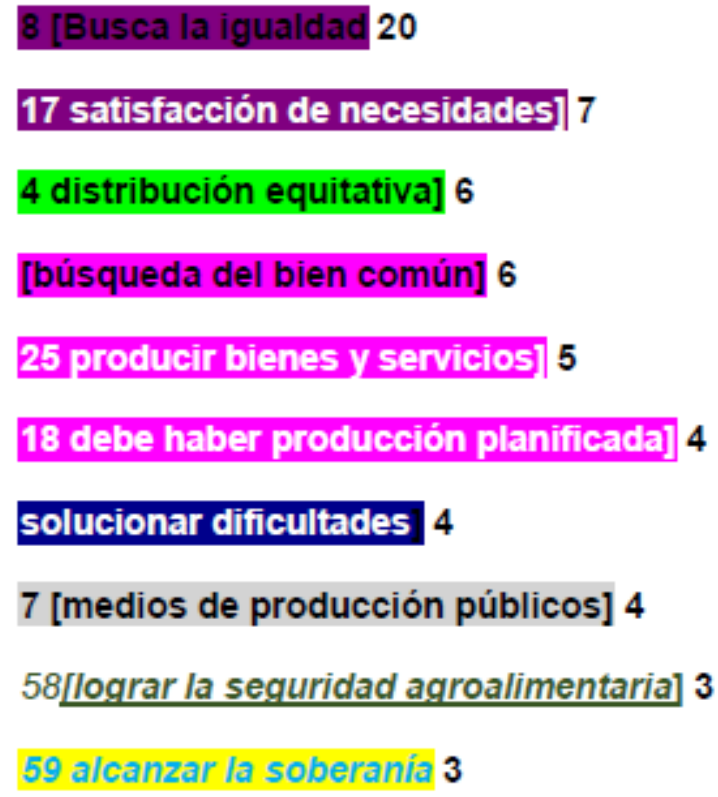




\section{Representación Social Inicial sobre Economía Socialista. (Miembros del Poder Popular Organizado)}

Figura 3 Representación Social Inicial sobre Economía Socialista (Miembros del Poder Popular Organizado)

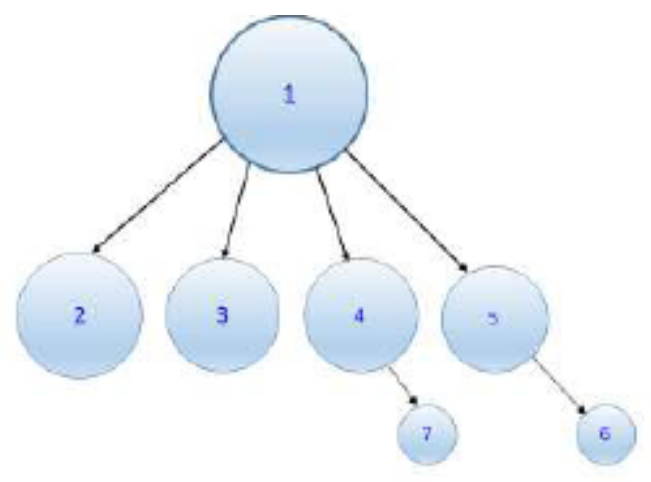

1. Busca la igualdad

2. Satisfacciones de necesidades

3. Distribución equitativa de los recursos

4. Búsquedas del bien común

5. Producir bienes y servicios

6. Debe haber producción planificada

7. Solucionar dificultades apremiantes

8. Medios de producción públicos

Es un proceso para la búsqueda de la igualdad, orientado a la satisfacción de las necesidades de las personas, priorizando la distribución equitativa de los recursos, para alcanzar el bien común, a través de la producción de bienes y servicios (fundamentalmente bienes primordiales) de manera planificada con medios de producción de propiedad pública que permitan solucionar las dificultades más apremiantes presentes en el pueblo

Nuevamente se advierte que no se hace alusión de manera concreta a la distribución equitativa del producto social o excedentes, ni al abandono de la división social del trabajo y no se explicita el carácter social de la propiedad pública, rasgos fundamentales de la Economía Socialista, ni a la relación armónica entre la economía y la madre tierra (Pachamama). En este caso, en el árbol de representación predomina de manera muy marcada la concepción referida a la búsqueda de la igualdad, resaltando su posicionamiento sobre todas las otras. 


\section{Nociones Iniciales Convergentes de la Economía Socialista}

Luego de haber sido elaboradas las representaciones sociales iniciales por separado de cada uno de los informantes clave (Profesionales, Funcionarios públicos y poder popular organizado), se consolido la información, integrando todas las intuiciones iniciales (cognemas) que emergieron de los discursos, lo cual permitió realizar un árbol de representación consolidado, que hemos denominado <Representación social convergente inicial de la Economía Socialista>. A continuación, se exponen las cognemas declarados:

8 [Busca la igualdad] $9+2+20=31$

17 satisfacción de necesidades] $11+11+7=29$

4 distribución equitativa] $10+7+6=23$

5 [búsqueda del bien común] 2+14+6 = 22

25 producir bienes y servicios $7+8+5=20$

11 [fomentar la participación] 8+9+1= 18

1 [idea de sistema] $4+10+2=16$

44 debe centrarse en el ser humano 15+1= 16

7 [medios de producción públicos] $8+2+4=14$

30 [propiciar el desarrollo endógeno-local] $5+7=12$

3 [medios de producción propiedad del Estado] 7+4= 11

45 mejorar la calidad de vida 9+2 = 11

2 sistema transitorio] $7+2=9$

14 solucionar dificultades $5+4=9$

47 modelo propio 9 


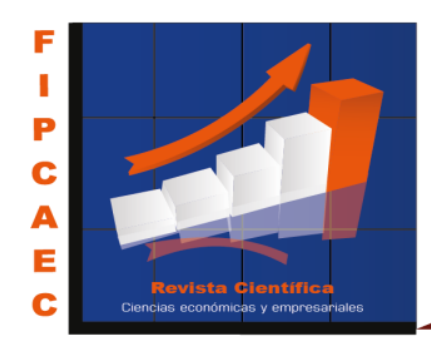

La Representación Social de la Economía Socialista en Venezuela

\section{Representación Social Inicial Convergente de la Economía Socialista}

Figura4. Representación Social Inicial convergente de la Economía Socialista

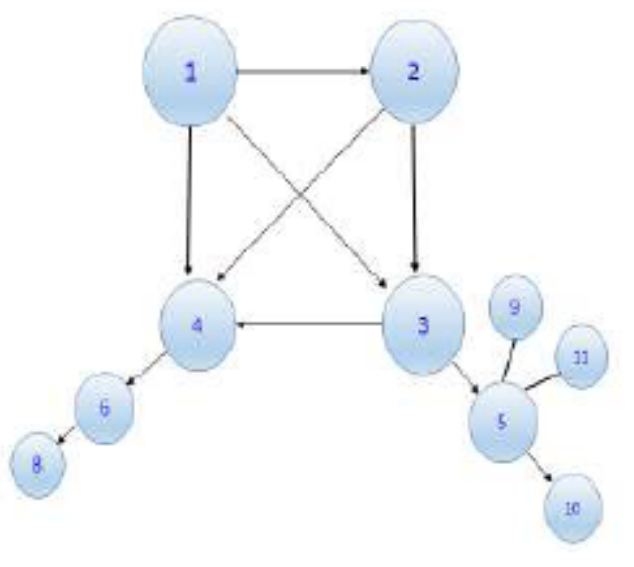
1. Busca la igualdad
2. Satisfacciones de necesidades
3. Distribución equitativa de los recursos
4. Búsquedas del bien común
5. Producir bienes y servicios
6. Fomentar la participación
7. Idea de sistema
8. Debe centrarse en el ser humano
9. Medios de producción públicos
10. Propiciar el desarrollo endógeno-local
11 Medios de producción del Estado

Se concibe la Economía Socialista como un proceso que tiene como propósito lograr la igualdad permitiendo la satisfacción de las necesidades de las personas, prevaleciendo la distribución equitativa de los recursos entre la población, con el fin de conseguir el bien común, por medio de la producción de los bienes y servicios necesarios en la sociedad, con medios de producción pudiendo ser de propiedad pública y/o propiedad del Estado. Se busca fomentar la participación, el proceso debe centrarse en el ser humano con la finalidad de consolidar un modelo de desarrollo endógeno-local. 
Se desprende del análisis de contenido efectuado en las entrevistas, que la referencia a la igualdad es solo declarativa y no sustantiva y la distribución equitativa no se hace de manera clara en función del producto social o excedentes, no hay del mismo modo expresiones concretas hacia el abandono de la división social del trabajo, entendida como la separación entre la toma de las decisiones fundamentales vinculadas al proceso productivo de parte de los dueños de los medios de producción y la exclusión en esa toma de las decisiones de los productores asociados, así como en la separación entre el trabajo intelectual y material, características fundamentales de la Economía Socialista.

Con respecto a lo relacionado con la propiedad de los medios de producción, se establece bajo la figura de propiedad estatal y/o de propiedad pública, ambas inclusive, se observa la idea de la necesidad de una nueva forma de propiedad de los medios de producción, sin embargo no se concibe la propiedad bajo la categoría de propiedad social de los medios de producción de manera directa, entendemos este aspecto como uno de los factores primordiales inherentes a la construcción del socialismo y su economía

Existe una manifestación relativamente pequeña respecto a la relación armónica que debe existir entre economía y la madre tierra (Pachamama). Estableciéndose la representación en función de cuatro cognemas principales (la igualdad, satisfacción de necesidades, distribución equitativa de los recursos y búsqueda del bien común).

\section{Expectativas por la Implementación de la Economía Socialista}

Para alcanzar el segundo objetivo propuesto en la investigación: Indagar que esperan los diversos actores sociales de la implementación de la economía socialista dentro del cambio epocal en Venezuela, utilizamos las informaciones derivadas de la guía de entrevista bajo el ítem 12, considerado como el más idóneo para dar respuesta a la segunda pregunta de investigación tal como se muestra en el siguiente cuadro: 


\section{$\left.\begin{array}{l}63 \text { preservar el medio ambiente } 4 \\ \mathbf{1 5} \text { [Equilibrio con la naturaleza] 2 }\end{array}\right\} 6$}

\section{9 [acabar con la burocracia] 5}

\section{2 [para poder convivir]5}

66 alcanzar la estabilidad social 4

\section{0 [Lograr la inclusión social] 4}

24 cambio de sistema 4

7 [medios de producción públicos] 4

49[combatir la impunidad] 4

[37 organización popular] 4

\section{1 niveles adecuados de producción 2}

[40 acabar con el consumismo] 2

\section{2establecer un nivel de consumo racional 2}

\section{0 alcanzar la estabilidad política 2}

Lo que se espera lograr por la implementación de la Economía Socialista, se refleja en el siguiente cuadro: 
A manera de síntesis podemos decir que lo que se espera de la Economía Socialista en primera instancia es la mejora en la calidad de vida de la población, el incremento del acceso a los Bienes y Servicios de toda índole, principalmente los bienes esenciales, el crecimiento de la producción, que el eje central de la actividad económica sea el ser humano, que se dé respuesta a la satisfacción de las necesidades de las personas, que se lleve a cabo la formación de un nuevo ciudadano con nuevos valores, que la acción gubernamental propenda a la búsqueda del bien común, así como a la distribución equitativa de los recursos y el alcanzar la estabilidad económica, vinculada a la preservación ambiental

\section{Discusión}

Las representaciones sociales iniciales de la economía socialista, son entendidas como un proceso que tiene como propósito primario lograr la igualdad, intención que a primera vista se percibe como muy plausible, no obstante, se aprecian ambigüedades e inconsistencias sobre su significado encontrándose en el discurso elementos que la refieren sin dar a conocer en que consiste esa igualdad, del mismo modo se entiende igualdad desde la perspectiva de igualdad en las oportunidades y en las condiciones de las personas, elemento clave en la construcción socialista, pero también se considera como igualdad la tenencia en términos de idénticas posesiones materiales.

Con respecto a la satisfacción de las necesidades, el énfasis se centra en las necesidades de tipo de material, necesidades cuya satisfacción es prioritaria y garantizan la propia existencia, a pesar de esto, la consideración hacia necesidades de otro tipo, como necesidades espirituales vinculadas a la idea de una vida con significado, de disponibilidad de tiempo libre, son elementos señalados en muy poca medida.

En igual forma, se resalta la idea de la distribución equitativa, muy vinculada a la noción de la igualdad, la idea presente en la distribución equitativa se percibe confusa, ambigua, en ocasiones se declara sin explicar en qué consiste, por momentos se vincula a la distribución de los recursos, principalmente materiales, inclusive se posiciona sobre la base que todo debe ser distribuido en partes iguales, este elemento contraviene los fines esenciales planteados en el socialismo y la Economía Socialista acerca de lo que debe ser la distribución y producción social de la vida.

\section{6}

FIPCAEC (núm. 6) Vol. 3, Año 3, Enero-Marzo 2018, pp. 86-111 
Relacionado al tema de la propiedad de los medios de producción, esta se establece bajo la figura de propiedad estatal y/o de propiedad pública, ambas inclusive, no se concibe la propiedad bajo la categoría de propiedad social de manera directa, sin embargo se observa la idea de la necesidad de una nueva forma de propiedad de los medios de producción, a nuestro modo de ver, este tema debe ser estudiado y trabajado de manera profunda en su significado, ya que entendemos este aspecto como uno de los factores primordiales inherentes a la construcción del socialismo y su economía.

En la configuración de esta representación, la cuestión del abandono de la división social del trabajo, entendida como la separación entre la toma de las decisiones fundamentales vinculadas al proceso productivo de parte de los dueños de los medios de producción, y su exclusión en esa toma de las decisiones de los productores asociados, así como en la separación entre el trabajo intelectual y material, no se visualiza en las manifestaciones de manera explícita.

En consecuencia, los aspectos descritos nos permiten decir que la representación social de la Economía Socialista se presenta en un nivel superficial, ya que algunas de las determinaciones esenciales que forman parte de la construcción socialista y de su economía no son referenciadas de manera precisa, entre ellas el abandono de la división social del trabajo, la superación de la dominación del valor de cambio sobre el valor de uso, la producción y distribución social de los excedentes, salvo lo concerniente a la relación armónica entre la economía y la naturaleza, que a pesar que se menciona de manera clara, la frecuencia de su aparición en el discurso no es la esperada, con respecto a la propiedad social de los medios de producción, se aprecia en el imaginario la visión sobre un nuevo modo de propiedad sobre los medios de producción, desde nuestra perspectiva estos conceptos no solo deben ser estudiados y entendidos en su totalidad, sino deben ser incorporados en la construcción y la práctica de la Economía Socialista ya que son parte esencial de su realización y como única vía posible.

En relación a lo que esperan los diversos actores sociales por la implementación de la economía socialista dentro del cambio epocal en Venezuela, la economía en general, y especialmente la Economía Socialista debe orientarse a proveer los satisfactores básicos para la sociedad, siendo esta la base de cualquier proceso revolucionario, si no existen satisfactorios básicos, ninguna 
acción o discurso por muy esperanzador que sea sirve, esta afirmación se corresponde con las expectativas creadas en torno a la implementación de la economía socialista dentro del cambio epocal en Venezuela, donde de acuerdo a las declaraciones dadas por los entrevistados primordialmente se destacan: (a) La mejora en la calidad de vida de la población. (b) El incremento al acceso a los Bienes y Servicios. (c) El crecimiento de la producción. (d) Que el eje central de la actividad económica sea el ser humano. (e) Que se dé respuesta a la satisfacción de las necesidades de las personas. (f) Que la acción gubernamental propenda a la búsqueda del bien común. (g) La distribución equitativa de los recursos. (h) El alcanzar la estabilidad económica. (i) La preservación del medio ambiente.

Cada una de estas expectativas se vinculan de manera estrecha a la acción de la actividad económica, que se encuentra en Venezuela en este momento histórico en su nivel de desempeño más bajo, entre otras razones debido a los procesos de desestabilización llevados a cabo en el país de manera reiterada, tanto en el en el pasado reciente, como en la actualidad, a la presencia de eventos como la guerra económica, el contrabando de extracción, políticas públicas en materia económica desacertadas, las interferencias e injerencia externa, el escaso nivel de productividad nacional, la dependencia de las importaciones, el descenso de los precios del petróleo, todas estas circunstancias han confluido para agravar esta situación.

Ahora bien, a nuestro entender, de continuar presentándose esta situación, donde no se proveen de los satisfactores básicos a la población, donde se evidencia una disminución en la calidad de vida de las personas que forman parte de esta sociedad, se coloca cada vez más cuesta arriba la construcción de la Economía Socialista, al mismo tiempo nos encontramos cerca de la posibilidad que se revierta el proceso de transformación que ha venido llevándose a cabo con la revolución bolivariana. 


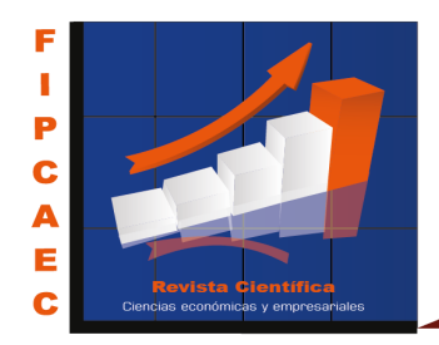

\section{Referencias}

1. Acosta, V. Fernández, C. San Vicente, I y otros. (2014). ¿Para qué sirve el capital? Un balance contemporáneo Obra principal de Karl Marx. 1ra edición. Editorial trinchera. Caracas.

2. Araya, S. (2002). Las representaciones sociales: Ejes teóricos para su discusión. CUADERNO DE CIENCIAS SOCIALES No 127.Facultad Latinoamericana de Ciencias Sociales (FLACSO) Sede Académica Costa Rica. San José.

3. Biardeau, J. (2007). ¿El proceso de transición hacia el nuevo Socialismo del siglo XXI?: un debate que apenas comienza, En Revista Venezolana de Economía y Ciencias Sociales, v.13 n.2 Caracas.

4. Carretero, E. (2001). Imaginarios sociales y crítica ideológica. Una perspectiva para la comprensión de la legitimación del orden social. Tesis de Doctorado. Universidad de Santiago de Compostela. España.

5. Foladori, G. (1990). Apuntes para una metodología materialista del análisis social. Trabajo y Capital. Ficha temática 1. Cuadernos de Extensión - N². Comisión Sectorial de Extensión y Actividades en el Medio (CSEAM). Montevideo.

6. Hernández, R. Fernández, C y Baptista, P (2006). Metodología de la investigación. Cuarta edición. McGraw-Hill Interamericana. México. Marx, K. (1973). El capital. Tomo III. FCE. México.

7. Marx, K. (1974). Miseria de la Filosofía. FCE. México.

8. Marx, K (2000). Critica al programa de Gotha. Ediciones elaleph.com

9. Marx y Engels. (1980). Obras Escogidas. Tomo I. Edición Progreso Moscú. Disponible en: http://bolchetvo.blogspot.com/. 
10. Marx y Engels. (1980). Obras Escogidas. Tomo III. Edición Progreso Moscú. Disponible en: http://bolchetvo.blogspot.com/.

11. Marx y Engels. (2007). La Ideología Alemana. Fundación Editorial el perro y la Rana. Caracas.

12. Mészáros, I. (2009) El desafío y la carga del tiempo histórico el socialismo del siglo XXI. Fundación Editorial el perro y la rana. Caracas.. Moscovici, S. (1979). El psicoanálisis, su imagen y su público. Huemul S.A. Buenos Aires.

13. Moscovici, S. (1993).Toward a Social Psychology of Science.En: Journal for the Theory of Social Behavior, 22(4):343-373.

14. Moscovici, S. (2000). Social representations.Explorations in Social Psychology.PolityPress, Cambridge.

15. Moscovici, S. (1991). La Sicología Social I. Barcelona, España: Paidós.

\section{References}

1. Acosta, V. Fernández, C. San Vicente, I and others. (2014). What is capital for? A contemporary balance Main work of Karl Marx. 1st edition Editorial trench. Caracas.

2. Araya, S. (2002). Social representations: Theoretical axes for discussion. SOCIAL SCIENCE NOTEBOOK No 127. Latin American Faculty of Social Sciences (FLACSO) Academic Headquarters Costa Rica. Saint Joseph.

3. Biardeau, J. (2007). The transition process towards the new Socialism of the 21 st century ?: a debate that is just beginning, In Venezuelan Journal of Economy and Social Sciences, v.13 n.2 Caracas.

4. Carretero, E. (2001). Social imaginary and ideological criticism. A perspective for the understanding of the legitimation of the social order. Doctoral thesis. University of Santiago de Compostela. Spain. 
5. Foladori, G. (1990). Notes for a materialistic methodology of social analysis. Labor and Capital Thematic file 1. Extension Notebooks - №2. Sectorial Commission of Extension and Activities in the Environment (CSEAM). Montevideo.

6. Hernández, R. Fernández, C and Baptista, P (2006). Investigation methodology. Fourth edition. McGraw-Hill Interamerican. Mexico. Marx, K. (1973). The capital Volume III FCE Mexico.

7. Marx, K. (1974). Misery of Philosophy. FCE Mexico.

8. Marx, K (2000). Criticize the Gotha program. Elaleph.com editions

9. Marx and Engels. (1980). Selected works. Volume I. Moscow Progress Edition. Available at: http://bolchetvo.blogspot.com/.

10. Marx and Engels. (1980). Selected works. Volume III Progress Edition Moscow. Available at: http://bolchetvo.blogspot.com/.

11. Marx and Engels. (2007). German Ideology Editorial Foundation the dog and the Frog. Caracas.

12. Mészáros, I. (2009) The challenge and burden of historical time the socialism of the 21 st century. Editorial Foundation the dog and the frog. Caracas.. Moscovici, S. (1979). Psychoanalysis, its image and its audience. Huemul S.A. Buenos Aires.

13. Moscovici, S. (1993) .Toward a Social Psychology of Science. In: Journal for the Theory of Social Behavior, 22 (4): 343-373.

14. Moscovici, S. (2000). Social representations.Explorations in Social Psychology.PolityPress, Cambridge.

15. Moscovici, S. (1991). Social Psychology I. Barcelona, Spain: Paidós.

(C2019 por el autor. Este artículo es de acceso abierto y distribuido según los términos y condiciones de la licencia Creative Commons Atribución-NoComercial-Compartirlgual 4.0 Internacional (CC BY-NC-SA 4.0) (https://creativecommons.org/licenses/by-nc-sa/4.0/). 